\title{
Study of $K^{0} \rightarrow \pi^{-} e^{+} \nu_{e} e^{+} e^{-}$in chiral perturbation theory
}

\author{
K. Tsuji and T. Sato \\ Department of Physics, Osaka University, \\ Toyonaka, Osaka 560-0043, Japan
}

(Dated: January 15, 2019)

\begin{abstract}
$K^{0} \rightarrow \pi^{-} e^{+} \nu_{e} e^{+} e^{-}$decay rates are studied up to the next-to-leading $\operatorname{order}\left(\mathcal{O}^{(4)}\right)$ in chiral perturbation theory. It is found that the $\mathcal{O}^{(4)}$ terms appreciably modify the shape of the invariant mass distribution of leptons $(3 e \nu)$ and the energy spectrum of neutrinos.
\end{abstract}

PACS numbers: 13.20.Eb, 12.39.Fe, 11.30.Rd, 12.40.Yx 


\section{INTRODUCTION}

The radiative semileptonic kaon decay, $K_{L} \rightarrow \pi^{ \pm} e^{\mp} \nu \gamma\left(K_{l 3 \gamma}\right)$ has been studied extensively [1, 2, 3, 4, 5, 6, 7, 8, 9, 10] within the chiral perturbation theory (ChPT)[11]. The amplitude of $K_{l 3 \gamma}$ can be written as a sum of an inner bremstrahlung(IB) amplitude and a structure dependent (SD) amplitude. The IB amplitude is the leading $\mathcal{O}\left(q^{-1}\right)$ and $\mathcal{O}\left(q^{0}\right)$ term of the photon momentum $(q)$ expansion of the amplitude and hence can be related to the nonradiative $K_{l 3}$ amplitude by using the theorems of Low [12] and Adler and Dothan[13]. On the other hand, the SD amplitude, which is of the order of $\mathcal{O}(q)$ and higher, contains new information on the hadron currents and therefore is the main interest for studying the $K_{L} \rightarrow \pi^{ \pm} e^{\mp} \nu \gamma\left(K_{l 3 \gamma}\right)$ reaction.

Fearing et al. [4] studied the radiative $K_{l 3}$ decay using the Low and Adler-Dothan theorems and the partial conservation of the axial current (PCAC) hypothesis. Later, Holstein [5] analyzed this decay process using the model independent ChPT at $\mathcal{O}\left(p^{4}\right)$ at tree level. The full $\mathcal{O}\left(p^{4}\right)$ ChPT analysis including the loop effects has been done by Bijnens et al. [6]. Further ChPT analysis of the $K_{e 3 \gamma}$ decay up to the $\mathcal{O}\left(p^{6}\right)$ terms was reported by Gasser et al. [7, 8]. In these calculations, the effect of the SD was found to be rather small in determining the integrated decay rate, but has appreciable effects on the differential decay rates. Sizable effects of the SD amplitude are found on the photon energy spectrum and pion energy distribution in the kinematic region where the experimental counting rate is small. The comparison of the data and a review of the theoretical studies on this reaction are given in Ref. [9] and Ref. [10], respectively.

In this paper, we report on a ChPT study of the semileptonic decay process of kaon $K^{0} \rightarrow$ $\pi^{-} e^{+} \nu_{e} e^{+} e^{-}\left(K_{e 3 e^{+} e^{-}}^{0}\right)$ process which differs from the $K_{L} \rightarrow \pi^{ \pm} e^{\mp} \nu \gamma\left(K_{l 3 \gamma}\right)$ discussed above because it involves the production of a timelike virtual photon followed by its decay into a $e^{+} e^{-}$pair. The recent $\mathrm{KTeV}$ experiment [14] is capable of measuring various differential decay rates of this reaction. The measured invariant mass distributions of $e^{+} e^{-}\left(M_{e^{+} e^{-}}\right)$ and leptons $\left(M_{3 e \nu}\right)$ and the energy spectrum of the neutrino will provide information for testing the ChPT predictions and extracting the information on the hadronic matrix elements. To analyze the data from this experiment and similar future experiments, it is necessary to have a full $\mathcal{O}\left(p^{4}\right)$ ChPT prediction of the differential decay rates of the $K_{e 3 e^{+} e^{-}}^{0}$ process. The purpose of this paper is to carry out such a calculation which, to our knowledge, is currently not available. We will examine various invariant mass distributions and energy spectrum. In particular we study which observables are more sensitive to the $\mathcal{O}\left(p^{4}\right)$ terms. We also predict the branching ratios of $K_{l 3 e^{+} e^{-}}^{0}(l=e, \mu)$ decay relative to $K_{l 3}^{0}$ decay. We however have not extracted the effects of the SD term and leave this more difficult problem for future investigations.

In section II, we summarize the effective Lagrangian employed in this work. The explicit form of the amplitudes of $K_{l 3 e^{+} e^{-}}^{0}(l=e, \mu)$ from ChPT up to $\mathcal{O}\left(p^{4}\right)$ are presented in

Section III. The invariant mass spectrum of the $K_{l 3 e^{+} e^{-}}^{0}(l=e, \mu)$ decay and effects of the $\mathcal{O}\left(p^{4}\right)$ amplitudes are discussed in section IV. 


\section{EFFECTIVE LAGRANGIAN}

Chiral perturbation theory is an effective field theory of QCD to describe low energy hadronic system using a systematic perturbation scheme. In this section, a standard effective Lagrangian of ChPT for Goldstone bosons [11, 15] is summarized for completeness. The dynamical variable of ChPT, $U(x)$, is parametrized by using octet fields $\phi_{a}$ of Goldstone bosons as

$$
U=\exp \left(i \frac{\phi(x)}{F_{0}}\right)
$$

with

$$
\phi(x)=\sum_{a=1}^{8} \lambda_{a} \phi_{a}(x)
$$

Here $F_{0}$ is the pion decay constant in the chiral limit and $\lambda_{a}$ are the Gell-Mann matrices. Following the standard counting rule [11, 15], the leading $\mathcal{O}\left(p^{2}\right)$ order chiral effective Lagrangian is given as

$$
\mathcal{L}^{(2)}=\frac{F_{0}^{2}}{4}<D_{\mu} U\left(D^{\mu} U\right)^{\dagger}+\chi U^{\dagger}+U \chi^{\dagger}>
$$

$<O>$ denotes the trace of the matrix $O . \quad \chi$ is given as $\chi=2 B_{0} M$ using the quark mass matrix $M=\operatorname{diag}\left(m_{u}, m_{d}, m_{s}\right)$ and parameter $B_{0}$. The covariant derivative $D_{\mu} U$, which includes a external electromagnetic field $\left(A_{\mu}\right)$ and the charged weak boson $\left(W_{\mu}\right)$, is defined as follows,

$$
\begin{aligned}
D_{\mu} U & =\partial_{\mu} U-i r_{\mu} U+i U l_{\mu}, \\
r_{\mu} & =v_{\mu}+a_{\mu}=-e Q A_{\mu}, \\
l_{\mu} & =v_{\mu}-a_{\mu}=-e Q A_{\mu}-\frac{g}{\sqrt{2}}\left(W_{\mu}^{+} T_{+}+\text {h.c. }\right) .
\end{aligned}
$$

$Q$ is the quark charge and $T_{+}$is given by the CKM matrix elements.

$$
Q=\frac{1}{3}\left(\begin{array}{ccc}
2 & 0 & 0 \\
0 & -1 & 0 \\
0 & 0 & -1
\end{array}\right), \quad T_{+}=\left(\begin{array}{ccc}
0 & V_{u d} & V_{u s} \\
0 & 0 & 0 \\
0 & 0 & 0
\end{array}\right)
$$

The next to-leading-order (NLO) $\mathcal{O}\left(p^{4}\right)$ effective Lagrangian following Ref. [11] is given as

$$
\begin{aligned}
\mathcal{L}^{(4)}= & L_{1}<D_{\mu} U\left(D^{\mu} U\right)^{\dagger}>^{2}+L_{2}<D_{\mu} U\left(D_{\nu} U\right)^{\dagger}><D^{\mu} U\left(D^{\nu} U\right)^{\dagger}> \\
& +L_{3}<D_{\mu} U\left(D^{\mu} U\right)^{\dagger} D_{\nu} U\left(D^{\nu} U\right)^{\dagger}>+L_{4}<D_{\mu} U\left(D^{\mu} U\right)^{\dagger}><\chi U^{\dagger}+U \chi^{\dagger}> \\
& +L_{5}<D_{\mu} U\left(D^{\mu} U\right)^{\dagger}\left(\chi U^{\dagger}+U \chi^{\dagger}\right)>+L_{6}<\chi U^{\dagger}+U \chi^{\dagger}>^{2} \\
& +L_{7}<\chi^{\dagger} U-U^{\dagger} \chi>^{2}+L_{8}<\chi U^{\dagger} \chi U^{\dagger}+U \chi^{\dagger} U \chi^{\dagger}> \\
& -i L_{9}<f_{\mu \nu}^{R} D^{\mu} U\left(D^{\nu} U\right)^{\dagger}+f_{\mu \nu}^{L} D^{\mu} D\left(D^{\nu} U\right)^{\dagger}>+L_{10}<U f_{\mu \nu}^{L} U^{\dagger} f_{R}^{\mu \nu}>,
\end{aligned}
$$

with

$$
f_{\mu \nu}^{R(L)}=\partial_{\mu} r(l)_{\nu}-\partial_{\nu} r(l)_{\mu}-i\left[r(l)_{\mu}, r(l)_{\nu}\right]
$$


and its filed tensors are defined as

$$
\begin{aligned}
f_{\mu \nu}^{R} & =\partial_{\mu} r_{\nu}-\partial_{\nu} r_{\mu}-i\left[r_{\mu}, r_{\nu}\right], \\
f_{\mu \nu}^{L} & =\partial_{\mu} l_{\nu}-\partial_{\nu} l_{\mu}-i\left[l_{\mu}, l_{\nu}\right] .
\end{aligned}
$$

The following piece of the chiral anomaly term [16, 17] contributes to the $\mathcal{O}\left(p^{4}\right)$ amplitude of $K_{e 3 e^{+} e^{-}}^{0}$ decay,

$$
\begin{aligned}
\mathcal{L}_{\text {anom }}^{(4)}= & -\frac{i e g}{16 \sqrt{2} \pi^{2}} \epsilon^{\mu \nu \rho \sigma} W_{\mu}^{+} \partial_{\nu} A_{\rho}\left\langle T_{+}\left\{\partial_{\sigma} U^{\dagger} Q U-2 U^{\dagger} \partial_{\sigma} U Q-2 Q U^{\dagger} \partial_{\sigma} U-U^{\dagger} Q \partial_{\sigma} U\right\}\right\rangle \\
& + \text { h.c.. }
\end{aligned}
$$

\section{LO AND NLO AMPLITUDES OF $K_{e 3 e^{+} e^{-}}$}

Using the chiral effective Lagrangian presented in the previous section, we study the amplitude of $K_{e 3 e^{+} e^{-}}^{0}$ decay up to the next-to-leading order.

$$
K^{0}\left(p_{1}\right) \rightarrow \pi^{-}\left(p_{2}\right)+e^{+}\left(k_{1}\right)+\nu_{e}\left(k_{2}\right)+e^{+}\left(k_{3}\right)+e^{-}\left(k_{4}\right) .
$$

The momentum of the virtual photon $q^{\mu}$ is given by $q^{\mu}=k_{3}^{\mu}+k_{4}^{\mu}$. The leading order amplitude $T^{(2)}$ is obtained from tree diagrams with vertices from $\mathcal{L}^{(2)}$. Loop diagrams from $\mathcal{L}^{(2)}$ and tree diagrams with vertices from $\mathcal{L}^{(4)}$ generate the next-to-leading order amplitude $T^{(4)}$.

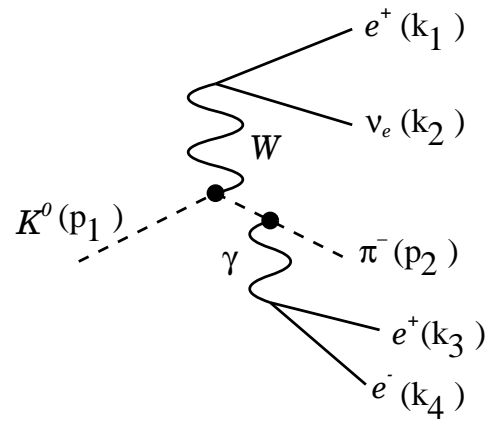

(a)

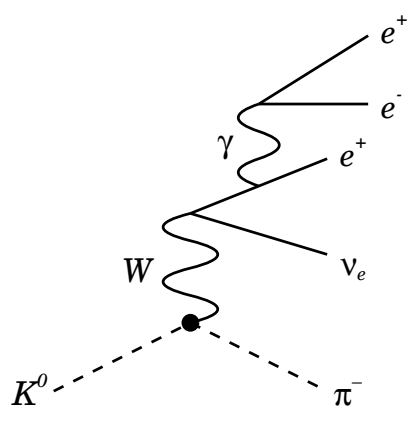

(b)

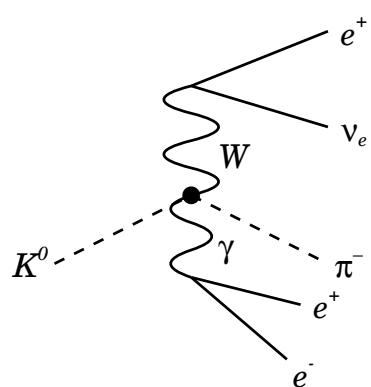

(c)

FIG. 1: The leading order diagrams contributing to the $K_{e 3 e^{+} e^{-}}^{0}$ decay. The dark circles are LO vertices from $\mathcal{L}^{(2)}$.

The leading order amplitude $T^{(2)}$ of the $K_{e 3 e^{+} e^{-}}^{0}$ decay shown in Fig. 1 is given as

$$
\begin{aligned}
T^{(2)}= & -\frac{G_{F}}{\sqrt{2}} e^{2} V_{u s}^{*} \frac{1}{q^{2}}\left[\bar{u}\left(k_{2}\right)\left\{g_{\mu \nu}-\frac{2 q_{\nu} p_{2 \mu}}{\left(p_{2}+q\right)^{2}-m_{\pi}^{2}}\right\} \gamma^{\nu}\left(1-\gamma_{5}\right) v\left(k_{1}\right)\right. \\
& \left.+\bar{u}\left(k_{2}\right)\left(\not p_{1}+\not p_{2}\right)\left(1-\gamma_{5}\right)\left\{\frac{2 k_{1 \mu}+\not \gamma_{\mu}}{\left(k_{1}+q\right)^{2}-m_{e}^{2}}-\frac{2 p_{2 \mu}}{\left(p_{2}+q\right)^{2}-m_{\pi}^{2}}\right\} v\left(k_{1}\right)\right] \bar{u}\left(k_{4}\right) \gamma^{\mu} v\left(k_{3}\right) .
\end{aligned}
$$


Here $G_{F}$ is Fermi constant and $V_{u s}$ is the CKM matrix element. Since we have two positrons in the final state, Eq. (14) represents the 'direct amplitude'. The 'exchange amplitude' is given from Eq. (14) by interchanging momentum and spins of the two positrons and by taking into account the phase $(-1)$. Eq. (14) satisfies gauge invariance and agrees with Eq. (5.12) of Ref. [6] when we replace the $e^{+} e^{-}$current by the photon polarization vector as

$$
\frac{e}{q^{2}} \bar{u}\left(k_{4}\right) \gamma^{\mu} v\left(k_{3}\right)=\epsilon^{* \mu}
$$

At the NLO, loop corrections and contributions of $\mathcal{L}^{(4)}$ are included. We take into account the diagrams shown in Fig. 2, They are the NLO correction of the pion(Fig. 2(a) ) and kaon(Fig. 2(b) electromagnetic form factors, $\pi K W$ vertex(Fig. 2(c), and Fig. 2(d)), $K \pi W \gamma$ vertex(Fig. 2(e) and anomaly term(Fig. 2(e) . The NLO T-matrix $\left(T^{(4)}\right)$ is given by the sum of six amplitudes as,

$$
T^{(4)}=T_{(a)}^{(4)}+T_{(b)}^{(4)}+T_{(c)}^{(4)}+T_{(d)}^{(4)}+T_{(e)}^{(4)}+T_{(e, a n o m)}^{(4)} .
$$

For completeness, the explicit forms of $T_{(i)}^{(4)}$ are described in the next subsections. It is worth noting that, using the formulas given in the Appendix $\mathrm{B}$, the expressions of $T^{(4)}$ can be shown to agree with those of Ref. [6] for the real photon limit.

\section{A. Pion and neutral kaon form factor}

In $K_{e 3 e^{+} e^{-}}^{0}$ reaction, the virtual photon momentum is non-zero and the NLO corrections to the pion and neutral kaon form factors contribute to the reaction amplitude. They are given by loop diagrams (Figs. 3(a), 3(b), 3(d) and $3(\mathrm{e})$ ) and vertices from $\mathcal{L}^{(4)}$ (Fig. $3(\mathrm{c})$. Only loop diagrams contribute to the neutral kaon form factor. The amplitudes $T_{(a)}^{(4)}$ and $T_{(b)}^{(4)}$ are given as

$T_{(a)}^{(4)}=-\frac{G_{F}}{\sqrt{2}} e^{2} V_{u s}^{*} \frac{H^{\pi}\left(q^{2}\right)}{q^{2}}\left[\bar{u}\left(k_{2}\right)\left(\not p_{1}+\not p_{2}+\not q\right)\left(1-\gamma_{5}\right) \frac{-2 p_{2 \mu}}{\left(p_{2}+q\right)^{2}-m_{\pi}^{2}} v\left(k_{1}\right)\right] \bar{u}\left(k_{4}\right) \gamma^{\mu} v\left(k_{3}\right)$,

$T_{(b)}^{(4)}=-\frac{G_{F}}{\sqrt{2}} e^{2} V_{u s}^{*} \frac{H^{K}\left(q^{2}\right)}{q^{2}}\left[\bar{u}\left(k_{2}\right)\left(\not p_{1}+\not p_{2}-\not q\right)\left(1-\gamma_{5}\right) \frac{2 p_{1 \mu}}{\left(p_{1}-q\right)^{2}-m_{K}^{2}} v\left(k_{1}\right)\right] \bar{u}\left(k_{4}\right) \gamma^{\mu} v\left(k_{3}\right)$.

Here we define $q=p_{1}-p_{2}$. The form factors $H^{\pi}$ and $H^{K}$ are given as

$$
\begin{aligned}
H^{\pi}\left(q^{2}\right) & =\frac{1}{F_{0}^{2}}\left[2 L_{9} q^{2}+A\left(m_{\pi}^{2}\right)+\frac{1}{2} A\left(m_{K}^{2}\right)-2 B_{22}\left(m_{\pi}^{2}, m_{\pi}^{2}, q^{2}\right)-B_{22}\left(m_{K}^{2}, m_{K}^{2}, q^{2}\right)\right] \\
H^{K}\left(q^{2}\right) & =\frac{1}{F_{0}^{2}}\left[\frac{1}{2} A\left(m_{K}^{2}\right)-\frac{1}{2} A\left(m_{\pi}^{2}\right)-B_{22}\left(m_{K}^{2}, m_{K}^{2}, q^{2}\right)+B_{22}\left(m_{\pi}^{2}, m_{\pi}^{2}, q^{2}\right)\right] .
\end{aligned}
$$

The functions $A\left(m^{2}\right)$ and $B_{22}\left(m_{1}^{2}, m_{2}^{2}, q^{2}\right)$ are given in the Appendix A. 


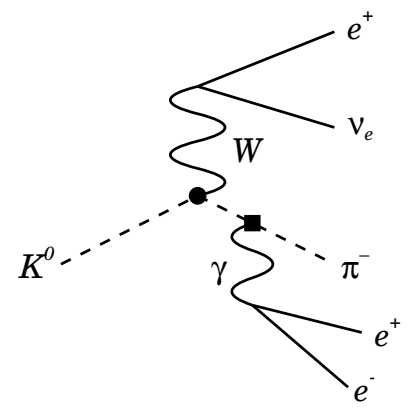

(a)

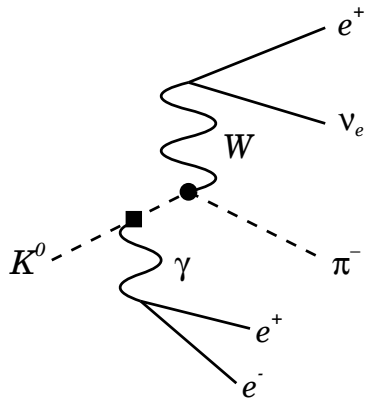

(b)

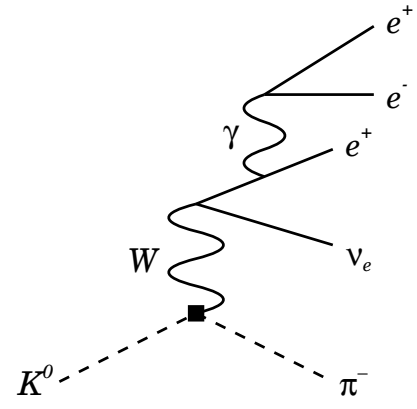

(c)

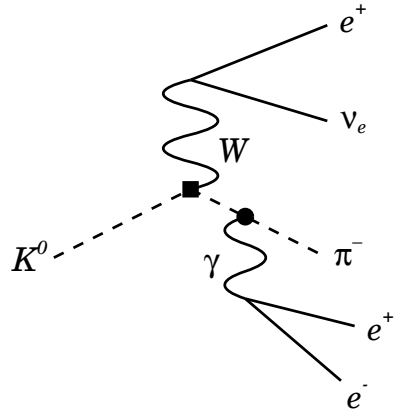

(d)

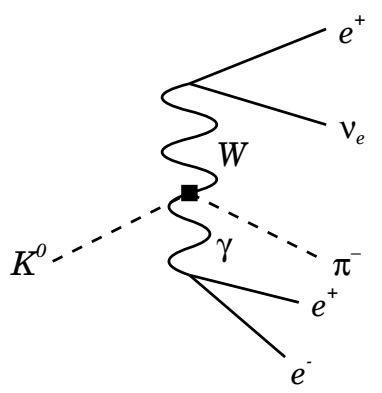

(e)

FIG. 2: The NLO diagrams contributing to the $K_{e 3 e^{+} e^{-}}^{0}$ decay. The dark squares(circles) are NLO (LO) vertices.

\section{B. $\pi K W$ vertices}

The diagrams contributing to the NLO $\pi K W$ vertex are shown in Fig. 4. The NLO amplitude $T_{(c)}^{(4)}$ and $T_{(d)}^{(4)}$ are given as

$$
\begin{aligned}
T_{(c)}^{(4)}= & -\frac{G_{F}}{\sqrt{2}} e^{2} V_{u s}^{*} \frac{1}{q^{2}}\left[\bar{u}\left(k_{2}\right)\left\{G_{1}\left(r_{c}, l_{c}\right)\left(\not p_{1}+\not p_{2}\right)+G_{2}\left(r_{c}, l_{c}\right)\left(\not p_{1}-\not p_{2}\right)\right\}\right. \\
& \left.\times\left(1-\gamma_{5}\right) \frac{2 k_{1 \mu}+\not \gamma_{\mu}}{\left(k_{1}+q\right)^{2}-m_{e}^{2}} v\left(k_{1}\right)\right] \bar{u}\left(k_{4}\right) \gamma^{\mu} v\left(k_{3}\right) \\
T_{(d)}^{(4)}= & \frac{G_{F}}{\sqrt{2}} e^{2} V_{u s}^{*} \frac{1}{q^{2}}\left[\bar{u}\left(k_{2}\right)\left\{G_{1}\left(r_{d}, l_{d}\right) \frac{\not p_{1}+\not p_{2}+\not q}{\left(p_{2}+q\right)^{2}-m_{\pi}^{2}}+G_{2}\left(r_{d}, l_{d}\right) \frac{\not p_{1}-\not p_{2}-\not 1}{\left(p_{2}+q\right)^{2}-m_{\pi}^{2}}\right\}\right. \\
& \left.\times\left(1-\gamma_{5}\right) v\left(k_{1}\right)\right] \bar{u}\left(k_{4}\right) 2 \not p_{2} v\left(k_{3}\right),
\end{aligned}
$$

with $r_{c}=p_{1}+p_{2}, l_{c}=p_{1}-p_{2}, r_{d}=p_{1}+p_{2}+q$ and $l_{d}=p_{1}-p_{2}-q$. Here $G_{1}$ and $G_{2}$ are the weak form factors of the $K \pi$ transition given as

$G_{1}(r, l)=\frac{2 L_{9}}{F_{0}^{2}} l^{2}+\frac{3}{8 F_{0}^{2}}\left\{A\left(m_{\eta}^{2}\right)+A\left(m_{\pi}^{2}\right)+2 A\left(m_{K}^{2}\right)\right\}-\frac{3}{2 F_{0}^{2}}\left\{B_{22}\left(m_{\pi}^{2}, m_{K}^{2}, l^{2}\right)+B_{22}\left(m_{K}^{2}, m_{\eta}^{2}, l^{2}\right)\right\}$, 


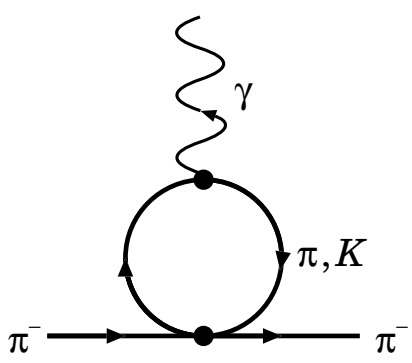

(a)

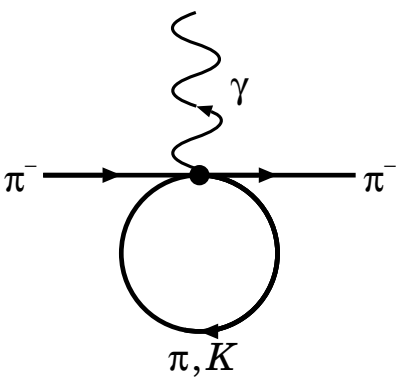

(b)

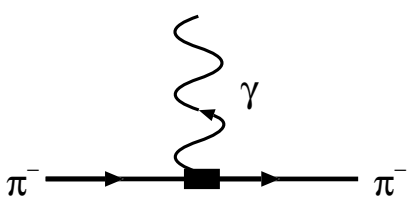

(c)

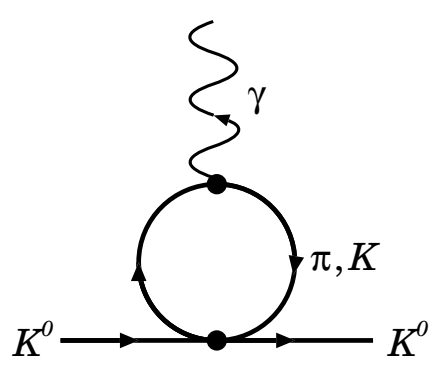

(d)

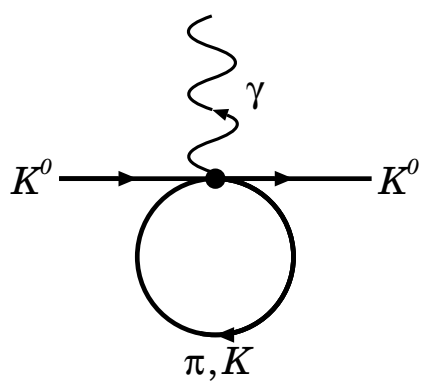

(e)

FIG. 3: The NLO diagrams contributing to the pion and the neutral kaon form factors. The dark box is NLO vertex from $\mathcal{L}^{(4)}$.

$$
\begin{aligned}
G_{2}(r, l)=\left[4\left(m_{K}^{2}-m_{\pi}^{2}\right) L_{5}-2 r \cdot l L_{9}+\frac{1}{2} A\left(m_{\eta}^{2}\right)-\frac{5}{12} A\left(m_{\pi}^{2}\right)+\frac{7}{12} A\left(m_{K}^{2}\right)\right. \\
+B\left(m_{\pi}^{2}, m_{K}^{2}, l^{2}\right)\left\{-\frac{1}{4} m_{\pi}^{2}+\frac{1}{6} m_{K}^{2}-\frac{5}{48} r^{2}+\frac{5}{16} l^{2}-\frac{3}{8} r \cdot l\right\} \\
+B\left(m_{K}, m_{\eta}^{2}, l^{2}\right)\left\{-\frac{1}{6} m_{\pi}^{2}-\frac{1}{12} m_{K}^{2}-\frac{1}{16} r^{2}+\frac{3}{16} l^{2}-\frac{3}{8} r \cdot l\right\} \\
+B_{1}\left(m_{\pi}^{2}, m_{K}^{2}, l^{2}\right)\left\{\frac{1}{2} m_{\pi}^{2}-\frac{1}{3} m_{K}^{2}+\frac{5}{24} r^{2}-\frac{5}{24} l^{2}+\frac{3}{2} r \cdot l\right\} \\
+B_{1}\left(m_{K}, m_{\eta}^{2}, l^{2}\right)\left\{\frac{1}{3} m_{\pi}^{2}+\frac{1}{6} m_{K}^{2}+\frac{1}{8} r^{2}-\frac{1}{8} l^{2}+\frac{3}{2} r \cdot l\right\} \\
+B_{21}\left(m_{\pi}^{2}, m_{K}^{2}, l^{2}\right)\left\{-\frac{5}{6} l^{2}-\frac{3}{2} r \cdot l\right\}+B\left(m_{K}, m_{\eta}^{2}, l^{2}\right)\left\{-\frac{3}{2} r \cdot l-\frac{1}{2} l^{2}\right\} \\
\left.-\frac{5}{6} B_{22}\left(m_{\pi}^{2}, m_{K}^{2}, l^{2}\right)-\frac{1}{2} B_{22}\left(m_{K}^{2}, m_{\eta}^{2}, l^{2}\right)\right] \frac{1}{F_{0}^{2}} .
\end{aligned}
$$

Those expressions agree with Eqs. (4.3)-(4.4) of [18] when the kaon and the pion are on the mass shell. 


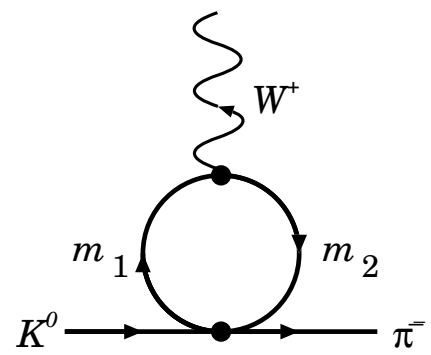

(a)

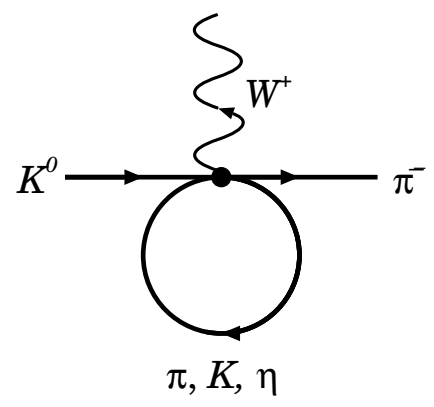

(b)

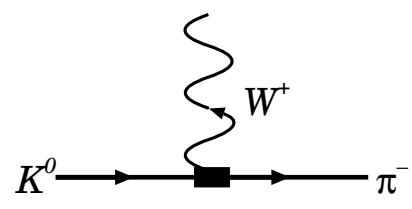

(c)

FIG. 4: The NLO diagrams contributing to $\pi K W$ vertices. Mesons $\left(m_{1}, m_{2}\right)$ are $\left(K^{+}, \eta\right)$, $\left(\pi^{+}, K^{0}\right),\left(K^{+}, \pi^{0}\right)$.

\section{C. $\pi K W \gamma$ vertices}

The amplitude with the NLO correction of $\pi K W \gamma$ vertex is expressed as

$$
T_{(e)}^{(4)}=-\frac{G_{F}}{\sqrt{2}} e^{2} V_{u s}^{*} \frac{1}{q^{2}} \bar{u}\left(k_{2}\right) \gamma_{\nu}\left(1-\gamma_{5}\right) v\left(k_{1}\right) \bar{u}\left(k_{4}\right) \gamma_{\mu} v\left(k_{3}\right) \sum_{\alpha=a}^{f} t_{(\alpha)}^{\mu \nu} \frac{1}{F_{0}^{2}} .
$$

The local interaction $\mathcal{L}^{(4)}$ shown in Fig. 5(a) gives

$$
\begin{aligned}
t_{(a)}^{\mu \nu}= & {\left[-\frac{1}{8} A\left(m_{\eta}^{2}\right)-\frac{11}{24} A\left(m_{\pi}^{2}\right)-\frac{5}{12} A\left(m_{K}^{2}\right)+4\left(m_{\pi}^{2}-m_{K}^{2}\right) L_{5}+4 L_{9}\left(W \cdot p_{1}-q \cdot p_{2}\right)\right.} \\
& \left.+4 L_{10} W \cdot q\right] g^{\mu \nu}+L_{9}\left[-4 W^{\mu} W^{\nu}-8 W^{\mu} p_{2}^{\nu}+8 p_{2}^{\mu} q^{\nu}+4 p_{2}^{\mu} W^{\nu}-4 q^{\mu} p_{2}^{\nu}\right] \\
& -4\left[L_{9}+L_{10}\right] W^{\mu} q^{\nu} .
\end{aligned}
$$

Here $q^{\mu}$ and $W^{\mu}$ are given as $q^{\mu}=k_{3}^{\mu}+k_{4}^{\mu}$ and $W^{\mu}=k_{1}^{\mu}+k_{2}^{\mu}$.

The contributions of the loop diagrams shown in Fig. $5(\mathrm{~b}), 5(\mathrm{f})$ are given as

$$
\begin{aligned}
t_{(b)}^{\mu \nu} & =\left[\frac{35}{12} A\left(m_{\pi}^{2}\right)+\frac{1}{4} A\left(m_{\eta}^{2}\right)+A\left(m_{K}^{2}\right)\right] g^{\mu \nu} \\
t_{(c)}^{\mu \nu} & =-\frac{10}{3} B_{22}\left(m_{\pi}^{2}, m_{\pi}^{2}, q^{2}\right) g^{\mu \nu} \\
t_{(d)}^{\mu \nu} & =\left[-\frac{1}{4} A\left(m_{K}^{2}\right)-2 B_{22}\left(m_{K}^{2}, m_{\eta}^{2}, W^{2}\right)-\frac{4}{3} B_{22}\left(m_{\pi}^{2}, m_{K}^{2}, W^{2}\right)\right] g^{\mu \nu} \\
& +\left[-2 B_{21}\left(m_{K}^{2}, m_{\eta}^{2}, W^{2}\right)+\frac{1}{2} B\left(m_{K}^{2}, m_{\pi}^{2}, W^{2}\right)-B_{1}\left(m_{K}^{2}, m_{\pi}^{2}, W^{2}\right)-\frac{4}{3} B_{21}\left(m_{\pi}^{2}, m_{K}^{2}, W^{2}\right)\right] W^{\mu} W^{\nu} \\
& +\left[2 B_{1}\left(m_{K}^{2}, m_{\eta}^{2}, W^{2}\right)+\frac{4}{3} B_{1}\left(m_{\pi}^{2}, m_{K}^{2}, W^{2}\right)\right]\left(p_{2}^{\mu}+2 W^{\mu}\right) W^{\nu} \\
& +\left[-\frac{1}{2} B\left(m_{K}^{2}, m_{\eta}^{2}, W^{2}\right)-\frac{1}{3} B\left(m_{\pi}^{2}, m_{K}^{2}, W^{2}\right)\right]\left(2 p_{2}^{\mu}+3 W^{\mu}\right) W^{\nu}
\end{aligned}
$$




\begin{tabular}{cccc}
\hline Coefficient & $K K \eta$ & $K K \pi$ & $\pi \pi K$ \\
\hline$a_{\beta}$ & $q \cdot\left(6 p_{2}+2 Q\right)$ & $2 p_{1} \cdot q$ & $q \cdot\left(6 p_{2}+2 Q\right)$ \\
$b_{\beta}$ & $Q \cdot\left(6 p_{2}+2 Q\right)$ & $2 p_{1} \cdot Q$ & $Q \cdot\left(6 p_{2}+2 Q\right)$ \\
$c_{\beta}$ & $-4 p_{2} \cdot Q-\frac{2}{3}\left(2 m_{\pi}^{2}+m_{K}^{2}\right)$ & $2\left(m_{\pi}^{2}-Q^{2}\right)$ & $-4 p_{2} \cdot Q$ \\
$d_{\beta}$ & 6 & 2 & 6 \\
$C\left(m_{1}^{2}, m_{2}^{2}, m_{3}^{2}\right)$ & $C\left(m_{K}^{2}, m_{K}^{2}, m_{\eta}^{2}\right)$ & $C\left(m_{K}^{2}, m_{K}^{2}, m_{\pi}^{2}\right)$ & $C\left(m_{\pi}^{2}, m_{\pi}^{2}, m_{K}^{2}\right)$ \\
\hline
\end{tabular}

TABLE I: Coefficients $a_{\beta}, b_{\beta}, c_{\beta}, d_{\beta}$ and the mass parameters of the functions $C_{2}, C_{i j}, C_{i j k}$.

$$
\begin{aligned}
t_{(e)}^{\mu \nu} & =\left[\frac{1}{2}\left(3 p_{2}+Q\right) \cdot Q B_{1}\left(m_{K}^{2}, m_{\eta}^{2}, Q^{2}\right)-\left\{p_{2} \cdot Q+\frac{1}{6}\left(m_{K}^{2}+2 m_{\pi}^{2}\right)\right\} B\left(m_{K}^{2}, m_{\eta}^{2}, Q^{2}\right)\right. \\
& +\frac{1}{4} A\left(m_{K}^{2}\right)-\frac{1}{6} A\left(m_{\pi}^{2}\right)+\left(\frac{1}{2} p_{1}+p_{2}+\frac{1}{3} Q\right) \cdot Q B_{1}\left(m_{\pi}^{2}, m_{K}^{2}, Q^{2}\right) \\
& \left.+\left(\frac{1}{2}\left(m_{\pi}^{2}-Q^{2}\right)-\frac{2}{3} p_{2} \cdot Q\right) B\left(m_{\pi}^{2}, m_{K}^{2}, Q^{2}\right)\right] g^{\mu \nu}, \\
t_{(f)}^{\mu \nu} & =\tilde{t}_{K K \eta}^{\mu \nu}+\tilde{t}_{K K \pi}^{\mu \nu}+\frac{2}{3}\left[g^{\mu \nu} B_{22}\left(m_{\pi}^{2}, m_{\pi}^{2}, q^{2}\right)+\tilde{t}_{\pi \pi K}^{\mu \nu}\right] .
\end{aligned}
$$

Here $Q^{\mu}=q^{\mu}+W^{\mu}$. $\tilde{t}_{\beta}^{\mu \nu}$ for $\beta=K K \eta, K K \pi, \pi \pi K$ is defined as

$$
\begin{aligned}
\tilde{t}_{\beta}^{\mu \nu}= & -g^{\mu \nu}\left[a_{\beta} C_{001}(\beta)+b_{\beta} C_{002}(\beta)+c_{\beta} C_{00}(\beta)\right]+p_{2}^{\mu} q^{\nu} d_{\beta}\left[C_{00}(\beta)-C_{001}(\beta)-C_{002}(\beta)\right] \\
+ & W^{\mu} q^{\nu}\left[-2 C_{001}(\beta)-4 C_{002}(\beta)-a_{\beta} C_{112}(\beta)-\left(a_{\beta}+b_{\beta}\right) C_{122}(\beta)\right. \\
& \left.-b_{\beta} C_{222}(\beta)+\left(b_{\beta}-c_{\beta}\right) C_{22}(\beta)+\left(a_{\beta}-c_{\beta}\right) C_{12}(\beta)+2 C_{00}(\beta)+c_{\beta} C_{2}(\beta)\right] \\
+ & W^{\mu} W^{\nu}\left[-4 C_{002}(\beta)-a_{\beta} C_{122}(\beta)-b_{\beta} C_{222}(\beta)+\left\{\frac{1}{2} b_{\beta}-c_{\beta}\right\} C_{22}(\beta)\right. \\
& \left.+\frac{1}{2} a_{\beta} C_{12}(\beta)+C_{00}(\beta)+\frac{1}{2} c_{\beta} C_{2}(\beta)\right] \\
- & W^{\mu} p_{2}^{\nu} d_{\beta} C_{002}(\beta)+p_{2}^{\mu} W^{\nu} d_{\beta}\left[-C_{002}(\beta)+\frac{1}{2} C_{00}(\beta)\right] .
\end{aligned}
$$

The coefficients $a_{\beta}, b_{\beta}, c_{\beta}, d_{\beta}$ and the mass parameters $m_{1}^{2}, m_{2}^{2}, m_{3}^{2}$ of the functions $C_{2}, C_{i j}, C_{i j k}$ defined in the Appendix $\mathrm{A}$ are given in table I.

\section{Chiral anomaly term}

Finally the contribution of the chiral anomaly term $T_{(e, \text { anom })}^{(4)}$ is given as

$$
T_{(e, \text { anom })}^{(4)}=-\frac{G_{F}}{\sqrt{2}} e^{2} V_{u s}^{*} \frac{1}{q^{2}}\left(-\frac{i}{8 \pi^{2} F_{0}^{2}}\right) \epsilon^{\mu \nu \rho \sigma} q_{\rho} W_{\sigma} \bar{u}\left(k_{2}\right) \gamma_{\nu}\left(1-\gamma_{5}\right) v\left(k_{1}\right) \bar{u}\left(k_{4}\right) \gamma_{\mu} v\left(k_{3}\right) \text {. }
$$




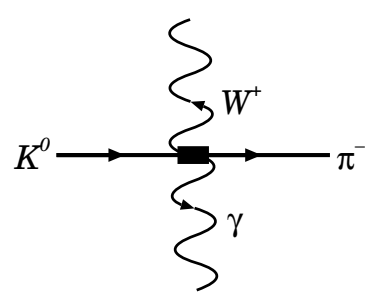

(a)

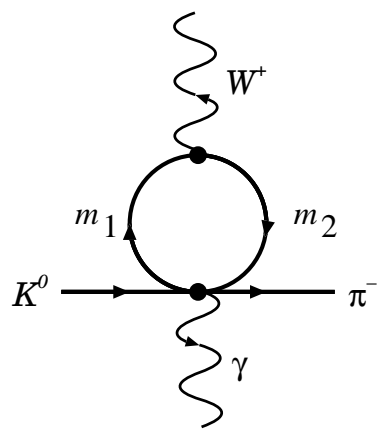

(d)

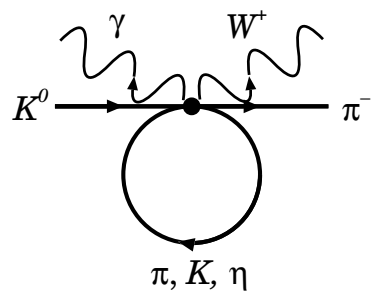

(b)

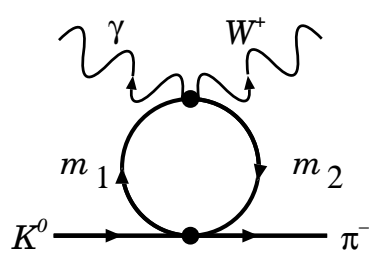

(e)

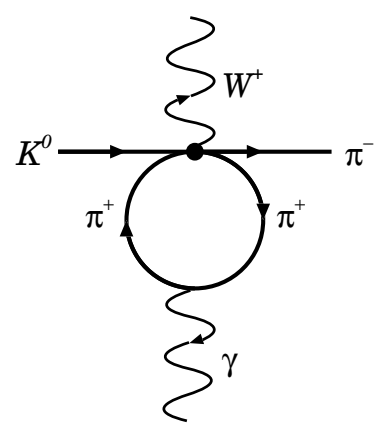

(c)

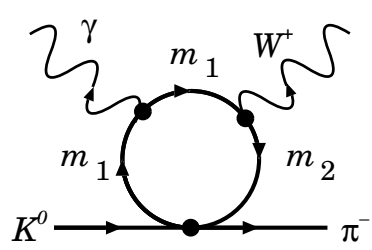

(f)

FIG. 5: The NLO diagrams contributing to $\pi K W \gamma$ vertexes. Mesons $\left(m_{1}, m_{2}\right)$ are $\left(K^{+}, \eta\right)$, $\left(\pi^{+}, K^{0}\right),\left(K^{+}, \pi^{0}\right)$.

\section{RESULTS AND DISCUSSIONS}

The total decay rate of $K_{e 3 e^{+} e^{-}}$is given by summing over the spins of the leptons in the final state:

$$
\Gamma\left(K_{e 3 e^{+} e^{-}}^{0}\right)=\frac{1}{2 m_{K}(2 \pi)^{11}} \int \frac{d \boldsymbol{p}_{2}}{2 p_{2}^{0}} \int \frac{d \boldsymbol{k}_{1}}{2 k_{1}^{0}} \cdots \int \frac{d \boldsymbol{k}_{4}}{2 k_{4}^{0}} \delta^{4}\left(p_{i}-p_{f}\right) \sum_{f}\left|T_{f i}\right|^{2} .
$$

The transition matrix element $T_{f i}$ is the sum of the LO amplitude $T^{(2)}$ and the NLO amplitude $T^{(4)}$.

The multi-dimensional phase space integration is performed by using the Vegas integration [19] method. One-loop integrals in $T^{(4)}$ are evaluated numerically using the package Looptools [20, 21]. In the following results, we use the masses of the neutral kaon and the charged pion and the charged pion decay constant [22],

$$
m_{K}=497.67 \mathrm{MeV}, \quad m_{\pi}=139.57 \mathrm{MeV}, \quad F_{\pi}=92.4 \mathrm{MeV} .
$$

We use the following low energy constants at the scale of $\mu=m_{\rho}=770 \mathrm{MeV}$ from Ref. [23],

$$
L_{9}^{r}\left(m_{\rho}\right)=6.9 \times 10^{-3}, \quad L_{10}^{r}\left(m_{\rho}\right)=-5.5 \times 10^{-3}
$$


and we use $F_{K} / F_{\pi}=1.22$ for $L_{5}$. The CKM matrix element and Fermi coupling constant are chosen as $\left|V_{u s}\right|=0.220[22]$ and $G_{F}=1.16637 \times 10^{-5} \mathrm{GeV}^{-2}$.

The role of the $\mathcal{O}\left(p^{4}\right)$ amplitude is studied for differential decay rates of $K_{e 3 e^{+} e^{-}}^{0}$. We examine the energy distribution of neutrino $\left(d \Gamma / d E_{\nu}\right)$, the invariant mass distribution of four leptons $e^{+} e^{-} e^{+} \nu_{e}\left(d \Gamma / d M_{3 e \nu}\right.$ with $\left.M_{3 e \nu}=\sqrt{\left(k_{1}+k_{2}+k_{3}+k_{4}\right)^{2}}=\sqrt{\left(p_{1}-p_{2}\right)^{2}}\right)$ and the invariant mass distribution of $e^{+} e^{-}\left(d \Gamma / d M_{e^{+} e^{-}}\right.$with $\left.M_{e^{+} e^{-}}=\sqrt{q^{2}}\right)$. The virtual photon momentum $q^{2}$ distribution is not available from $K_{l 3 \gamma}^{0}$ decay. The calculated invariant mass distributions $d \Gamma / d M_{3 e \nu_{e}}, d \Gamma / E_{\nu}$ and $d \Gamma / M_{e^{+} e^{-}}$are shown in Fig. 6] with the $\mathcal{O}\left(p^{2}\right)$ amplitude (dash curve) and the $\mathcal{O}\left(p^{2}\right)+\mathcal{O}\left(p^{4}\right)$ amplitude (solid curve). In those invariant mass distributions, the second term of Eq.(14) in the LO amplitude plays a dominant role. Around the peak of those distributions, effects of the $\mathcal{O}\left(p^{4}\right)$ amplitude contribute about $10 \%$ of the $M_{3 e \nu_{e}}$ and $E_{\nu}$ distributions. A smaller effect of the NLO amplitude is found for the $M_{e^{+} e^{-}}$distribution.

The effect of the $\mathcal{O}\left(p^{4}\right)$ amplitude on the shape of the mass distributions can be more clearly seen in the ratio $d \Gamma(L O+N L O) / d \Gamma(L O)$. Those ratios are shown in Fig. 7, The solid curves show results using the full $\mathcal{O}\left(p^{4}\right)$ amplitudes, while the dashed curves show results including only loop contributions. In the dashed curves, the $\mathcal{O}\left(p^{4}\right)$ amplitudes are calculated with $L_{5}=L_{10}=0$ (dash-dot), i.e. only $L_{9}$ is included in addition to loop contributions and the anomaly term. The $\mathcal{O}\left(p^{4}\right)$ effects increase with energy for $M_{3 e \nu_{e}}$ and neutrino energy distributions. They become about 1.2 to 1.25 for $M_{3 e \nu_{e}}>200 \mathrm{MeV}$ and about $10 \%$ around the peak of the $E_{\nu}$ distribution. For both $M_{3 e \nu_{e}}$ and $E_{\nu}$ distributions, it will be possible to test the $\mathcal{O}\left(p^{4}\right)$ effects in the energy region where the decay rates themselves are large. As far as the effects of the low energy constants are concerned, the most important contribution is $L_{9}$. The effects of $L_{5}, L_{10}$ and the chiral anomaly are found to be small for $M_{3 e \nu_{e}}$ and $E_{\nu}$ distributions. The loops effects reduce the invariant mass distributions by about $5 \%$. The shape of the invariant mass distributions is almost not affected by the loop diagrams. In $M_{e^{+} e^{-}}$, the effects of the $\mathcal{O}\left(p^{4}\right)$ terms appear in a slightly different way. The $L_{9}$ term mainly contributes to the $M_{e^{+} e^{-}}$distribution below $M_{e^{+} e^{-}}=100 \mathrm{MeV}$. Above $150 \mathrm{MeV}, L_{10}$ begins to contribute and tends to cancel the contribution of $L_{9}$. The matrix element of the chiral anomaly term is proportional to $\epsilon^{\mu \nu \rho \sigma} q_{\rho} W_{\sigma}$, and the amplitude is directly proportional to $M_{e^{+} e^{-}}=\sqrt{q^{2}}$. The effects of the chiral anomaly term start to be sizable above $M_{e^{+} e^{-}}=150 \sim 200 \mathrm{MeV}$ and the $M_{e^{+} e^{-}}$distribution in the high energy region will be interesting even if the decay rate is quite small. In this energy region, the relative importance of the 'exchange' amplitude compared with the 'direct' amplitude increases because of the photon propagator. A straightforward interpretation of the $q^{2}$ dependence of the $M_{e^{+} e^{-}}$distribution may be possible for $K_{\mu 3 e^{+} e^{-}}^{0}$ decay, which is free from the exchange effects.

Finally we examine the total decay rate of $K_{l 3 e^{+} e^{-}}^{0}(l=e, \mu)$ relative to the $K_{l 3}^{0}$ decay rate as

$$
\mathcal{R}\left(K_{l 3 e^{+} e^{-}}^{0}\right)=\frac{\Gamma\left(K_{l 3 e^{+} e^{-}}^{0}\right)}{\Gamma\left(K_{l 3}^{0}\right)} . \quad(l=e, \mu)
$$

The decay rate $\Gamma\left(K_{l 3}^{0}\right)$ is calculated in ChPT up to $\mathcal{O}\left(p^{4}\right)$. Here the ratios are calculated in the absence of real and virtual photon corrections [7]. Our results on $\mathcal{R}$ are shown in 
Table II for three cases using the full $\mathcal{O}\left(p^{4}\right)$ amplitude, the $\mathcal{O}\left(p^{2}\right)$ amplitude, and loop corrections. In the last case, we just set $L_{i}^{r}=0$. Including the $\mathcal{O}\left(p^{4}\right)$ amplitudes, the total decay rate is increased by $6 \%(8 \%)$ for the $K_{e 3 e^{+} e^{-}}^{0}\left(K_{\mu 3 e^{+} e^{-}}^{0}\right)$ decay. The loop correction reduces the decay rate, which is consistent with the one reported in [6] for $K_{l 3 \gamma}$. The effects of the chiral anomaly are very small on the total decay rate.

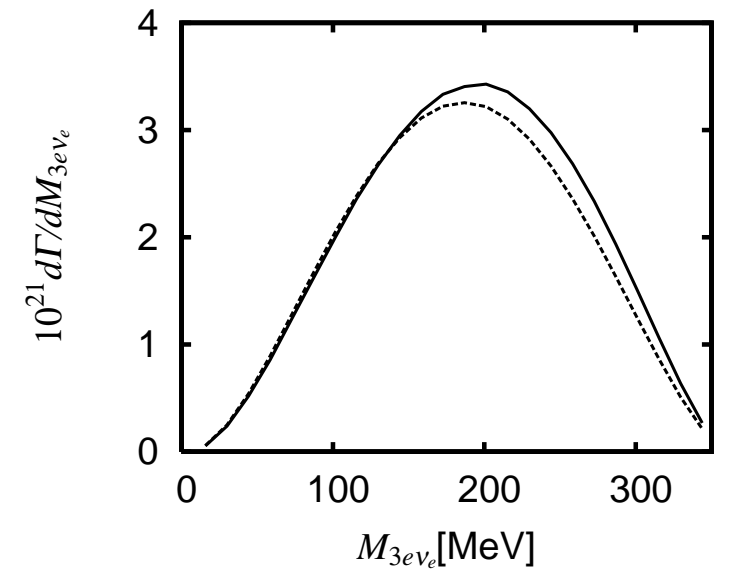

(a)

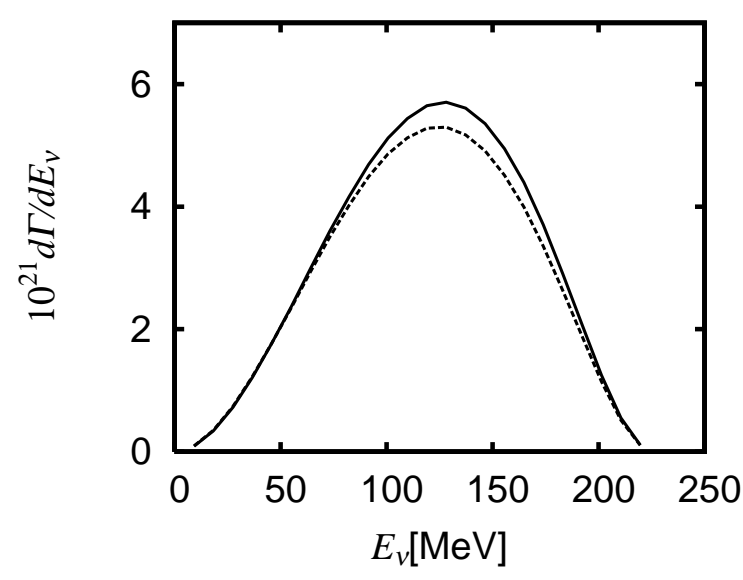

(b)

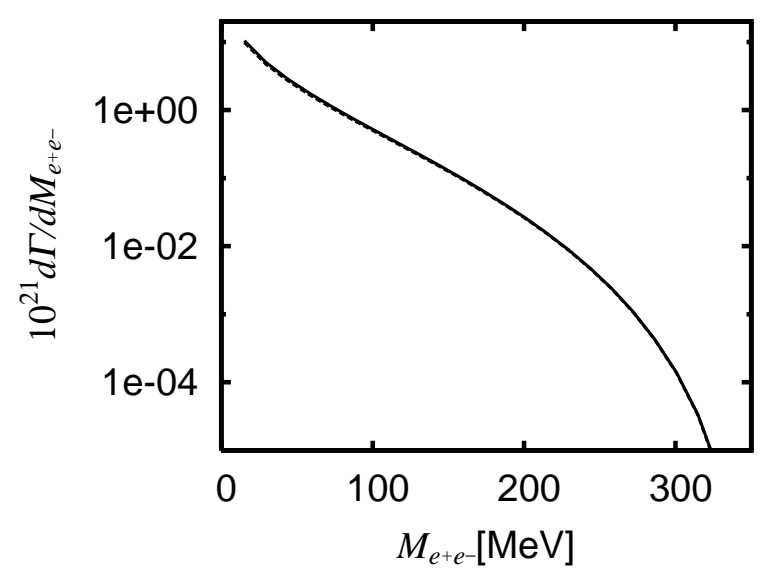

(c)

FIG. 6: $\quad M_{3 e \nu_{e}}, E_{\nu}$, and $M_{e^{+} e^{-}}$distributions of $K_{e 3 e^{+} e^{-}}^{0}$ decay. The differential decay rates are calculated with $\mathcal{O}\left(p^{2}\right)$ ( dashed curve) and and $\mathcal{O}\left(p^{2}\right)+\mathcal{O}\left(p^{4}\right)$ (solid curve). 


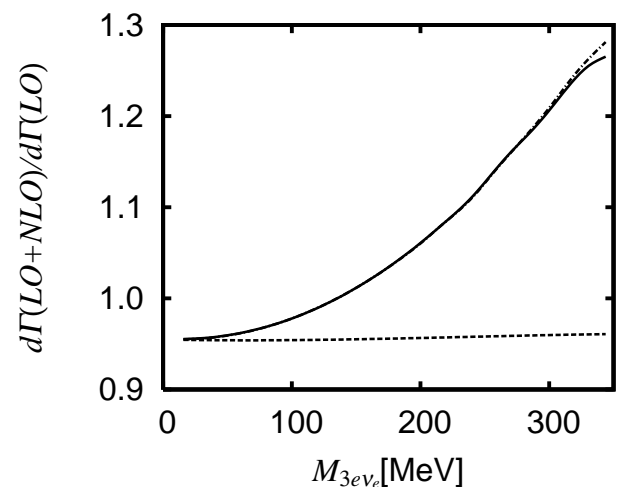

(a)

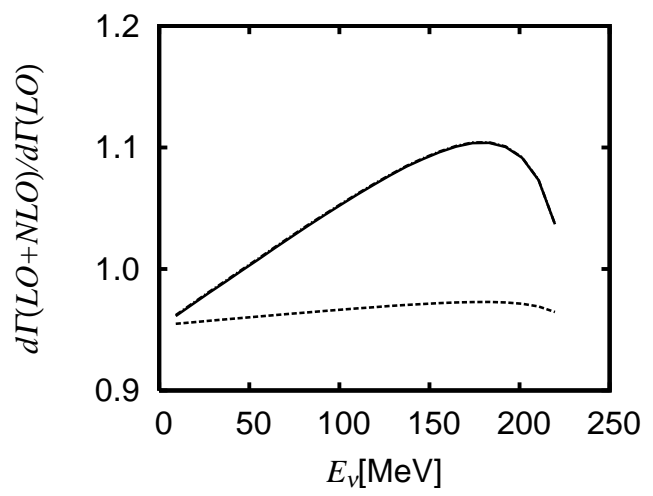

(b)

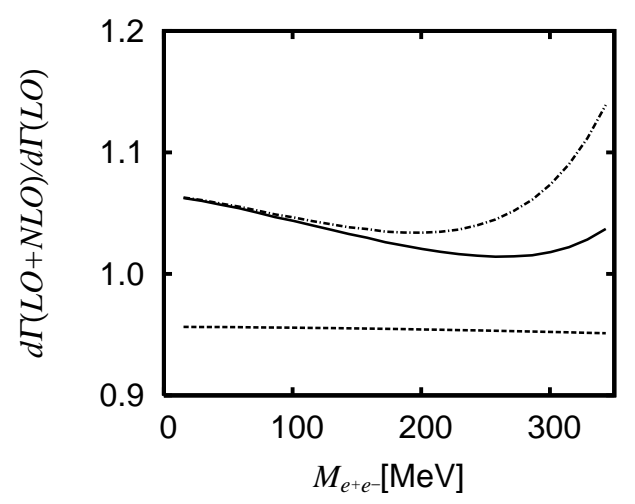

(c)

FIG. 7: The ratio of the $L O+N L O$ to the $L O$ for $M_{3 e \nu_{e}}, E_{\nu}$ and $M_{e^{+} e^{-}}$distributions. The solid, dash-dot and dotted curves show results with full $\mathcal{O}\left(p^{4}\right), \mathcal{O}\left(p^{4}\right)$ with $L_{5}^{r}=L_{10}^{r}=0$ and loop effects.

\begin{tabular}{cll}
\hline & $\mathcal{R}\left(K_{e 3 e^{+} e^{-}}^{0}\right)$ & $\mathcal{R}\left(K_{\mu 3 e^{+} e^{-}}^{0}\right)$ \\
\hline full $\mathcal{O}\left(p^{4}\right)$ & $1.34 \times 10^{-4}$ & $3.50 \times 10^{-4}$ \\
tree level & $1.26 \times 10^{-4}$ & $3.24 \times 10^{-4}$ \\
only loops at $\mathcal{O}\left(p^{4}\right)$ & $1.20 \times 10^{-4}$ & $3.12 \times 10^{-4}$ \\
\hline
\end{tabular}

TABLE II: The ratio of the branching ratios of $K_{e 3 e^{+} e^{-}}^{0}\left(K_{\mu 3 e^{+} e^{-}}^{0}\right)$ decay to $K_{e 3}\left(K_{\mu 3}\right)$ decay.

\section{SUMMARY}

In summary, we have studied the differential decay rates of $K_{e 3 e^{+} e^{-}}$in $\mathrm{ChPT}$ up to $\mathcal{O}\left(p^{4}\right)$ for the first time and found that the $M_{3 e \nu_{e}}$ and $E_{\nu}$ distributions will be suitable observables to test the $\mathcal{O}\left(p^{4}\right)$ amplitudes. Our analysis will provide the first hint to analyze the 
various mass distributions of the $K_{e 3 e^{+} e^{-}}^{0}$ decay. The data of $K_{e 3 e^{+} e^{-}}^{0}$ from $\mathrm{KTeV}$ has been analyzed using the results obtained in this work and it was found that the NLO calculation consistently improves that of the LO one[14]. Once the precise data of the $K_{e 3 e^{+} e^{-}}^{0}$ decay are available, the next task is to separate the IB and SD contribution in this process and obtain new information from this decay mode.

\section{Acknowledgments}

The authors would like to thank Prof. T. Yamanaka and Dr. K. Kotera for many useful suggestions on the analysis of $\mathrm{KTeV}$ data. We also thank Prof. K. Kubodera and Drs. T. -S. H. Lee and B. Julia-Diaz for discussions. KT was supported by the 21st Century COE Program named "Towards a New Basic Science: Depth and Synthesis".

\section{APPENDIX A: LOOP INTEGRALS}

Functions $A, B_{i}, C_{i}$ are defined as follows.

$$
\begin{aligned}
& A\left(m_{1}^{2}\right)=\frac{\mu^{4-n}}{i} \int \frac{d^{n} q}{(2 \pi)^{n}} \frac{1}{q^{2}-m_{1}^{2}}, \\
& B\left(m_{1}^{2}, m_{2}^{2}, p^{2}\right)=\frac{\mu^{4-n}}{i} \int \frac{d^{n} q}{(2 \pi)^{n}} \frac{1}{\left(q^{2}-m_{1}^{2}\right)\left((q-p)^{2}-m_{2}^{2}\right)}, \\
& B_{\mu}\left(m_{1}^{2}, m_{2}^{2}, p^{2}\right)=\frac{\mu^{4-n}}{i} \int \frac{d^{n} q}{(2 \pi)^{n}} \frac{q_{\mu}}{\left(q^{2}-m_{1}^{2}\right)\left((q-p)^{2}-m_{2}^{2}\right)}=p_{\mu} B_{1}\left(m_{1}^{2}, m_{2}^{2}, p^{2}\right), \\
& B_{\mu \nu}\left(m_{1}^{2}, m_{2}^{2}, p^{2}\right)=\frac{\mu^{4-n}}{i} \int \frac{d^{n} q}{(2 \pi)^{n}} \frac{q_{\mu} q_{\nu}}{\left(q^{2}-m_{1}^{2}\right)\left((q-p)^{2}-m_{2}^{2}\right)} \\
& =p_{\mu} p_{\nu} B_{21}\left(m_{1}^{2}, m_{2}^{2}, p^{2}\right)+g_{\mu \nu} B_{22}\left(m_{1}^{2}, m_{2}^{2}, p^{2}\right) \text {, } \\
& B_{\mu \nu \alpha}\left(m_{1}^{2}, m_{2}^{2}, p^{2}\right)=\frac{\mu^{4-n}}{i} \int \frac{d^{n} q}{(2 \pi)^{n}} \frac{q_{\mu} q_{\nu} q_{\alpha}}{\left(q^{2}-m_{1}^{2}\right)\left((q-p)^{2}-m_{2}^{2}\right)} \\
& =p_{\mu} p_{\nu} p_{\alpha} B_{31}\left(m_{1}^{2}, m_{2}^{2}, p^{2}\right)+\left(p_{\mu} g_{\nu \alpha}+p_{\nu} g_{\mu \alpha}+p_{\alpha} g_{\mu \nu}\right) B_{32}\left(m_{1}^{2}, m_{2}^{2}, p^{2}\right), \\
& C\left(m_{1}^{2}, m_{2}^{2}, m_{3}^{2}, q^{2}, W^{2}, Q^{2}\right)=\frac{\mu^{4-n}}{i} \int \frac{d^{n} k}{(2 \pi)^{n}} \frac{1}{k^{2}-m_{1}^{2}} \frac{1}{(k-q)^{2}-m_{2}^{2}} \frac{1}{(k-Q)^{2}-m_{3}^{2}} \\
& C_{\mu}\left(m_{1}^{2}, m_{2}^{2}, m_{3}^{2}, q^{2}, W^{2}, Q^{2}\right)=\frac{\mu^{4-n}}{i} \int \frac{d^{n} k}{(2 \pi)^{n}} \frac{1}{k^{2}-m_{1}^{2}} \frac{1}{(k-q)^{2}-m_{2}^{2}} \frac{1}{(k-Q)^{2}-m_{3}^{2}} k^{\mu} \\
& =q_{\mu} C_{1}+Q_{\mu} C_{2} \text {, } \\
& C_{\mu \nu}\left(m_{1}^{2}, m_{2}^{2}, m_{3}^{2}, q^{2}, W^{2}, Q^{2}\right)=\frac{\mu^{4-n}}{i} \int \frac{d^{n} k}{(2 \pi)^{n}} \frac{1}{k^{2}-m_{1}^{2}} \frac{1}{(k-q)^{2}-m_{2}^{2}} \frac{1}{(k-Q)^{2}-m_{3}^{2}} k^{\mu} k^{\nu}
\end{aligned}
$$




$$
\begin{aligned}
= & g_{\mu \nu} C_{00}+q_{\mu} q_{\nu} C_{11}+Q_{\mu} Q_{\nu} C_{22}+\left(q_{\mu} Q_{\nu}+Q_{\mu} q_{\nu}\right) C_{12}, \quad \text { (A8) } \\
C_{\mu \nu \rho}\left(m_{1}^{2}, m_{2}^{2}, m_{3}^{2}, q^{2}, W^{2}, Q^{2}\right)= & \frac{\mu^{4-n}}{i} \int \frac{d^{n} k}{(2 \pi)^{n}} \frac{1}{k^{2}-m_{1}^{2}} \frac{1}{(k-q)^{2}-m_{2}^{2}} \frac{1}{(k-Q)^{2}-m_{3}^{2}} k^{\mu} k^{\nu} k^{\rho} \\
= & \left(g_{\mu \nu} q_{\rho}+g_{\nu \rho} q_{\mu}+g_{\mu \rho} q_{\nu}\right) C_{001}+\left(g_{\mu \nu} Q_{\rho}+g_{\nu \rho} Q_{\mu}+g_{\mu \rho} Q_{\nu}\right) C_{002} \\
& +\left(q_{\mu} q_{\nu} Q_{\rho}+q_{\mu} Q_{\nu} q_{\rho}+Q_{\mu} q_{\nu} q_{\rho}\right) C_{112} \\
& +\left(Q_{\mu} Q_{\nu} q_{\rho}+Q_{\mu} q_{\nu} Q_{\rho}+q_{\mu} Q_{\nu} Q_{\rho}\right) C_{122} \\
& +q_{\mu} q_{\nu} q_{\rho} C_{111}+Q_{\mu} Q_{\nu} Q_{\rho} C_{222} .
\end{aligned}
$$

Here $\epsilon=4-n, Q^{\mu}=q^{\mu}+W^{\mu}$.

\section{APPENDIX B: COMPARISON WITH THE CHPT CALCULATION OF $K_{l 3 \gamma}$ RE- ACTION}

In the real photon limit $q^{2}=0$, one can show that our formula for the NLO amplitudes of $K_{e 3 e^{+} e^{-}}^{0}$ agrees with the amplitudes of $K_{l 3 \gamma}$ given in Ref. [6] by using the following relations.

$$
\begin{aligned}
& A\left(m_{1}^{2}\right)= \frac{m_{1}^{2}}{16 \pi^{2}} \lambda_{0}+\bar{A}\left(m_{1}^{2}\right), \\
& B\left(m_{1}^{2}, m_{2}^{2}, p^{2}\right)= \frac{\lambda_{0}}{16 \pi^{2}}+\bar{B}\left(m_{1}^{2}, m_{2}^{2}, p^{2}\right), \\
& B_{1}\left(m_{1}^{2},, m_{2}^{2}, p^{2}\right)= \frac{\lambda_{0}}{32 \pi^{2}}+\frac{1}{2 p^{2}}\left\{\bar{A}\left(m_{2}^{2}\right)-\bar{A}\left(m_{1}^{2}\right)+\left(m_{1}^{2}-m_{2}^{2}+p^{2}\right) \bar{B}\left(m_{1}^{2}, m_{2}^{2}, p^{2}\right)\right\}, \\
& B_{22}\left(m_{1}^{2}, m_{2}^{2}, p^{2}\right)= \frac{\lambda_{0}}{64 \pi^{2}}\left(m_{1}^{2}+m_{2}^{2}-\frac{p^{2}}{3}\right)+\frac{1}{96 \pi^{2}}\left(m_{1}^{2}+m_{2}^{2}-\frac{p^{2}}{3}\right) \\
&+\frac{1}{6} \bar{A}\left(m_{2}^{2}\right)+\frac{m_{1}^{2}}{3} \bar{B}\left(m_{1}^{2}, m_{2}^{2}, p^{2}\right)-\frac{1}{6}\left(p^{2}+m_{1}^{2}-m_{2}^{2}\right) \bar{B}_{1}\left(m_{1}^{2}, m_{2}^{2}, p^{2}\right), \text { (B4) } \\
&+\frac{1}{3 p^{2}} \bar{A}\left(m_{2}^{2}\right)-\frac{m_{1}^{2}}{3 p^{2}} \bar{B}\left(m_{1}^{2}, m_{2}^{2}, p^{2}\right)+\frac{2}{3 p^{2}}\left(p^{2}+m_{1}^{2}-m_{2}^{2}\right) \bar{B}_{1}\left(m_{1}^{2}, m_{2}^{2}, p^{2}\right), \\
& B_{21}\left(m_{1}^{2}, m_{2}^{2}, p^{2}\right)= \frac{1}{48 \pi^{2}}-\frac{1}{96 \pi^{2} p^{2}}\left(m_{1}^{2}+m_{2}^{2}-\frac{p^{2}}{3}\right) \\
& \bar{A}\left(m_{1}^{2}\right)=-\frac{m_{1}^{2}}{16 \pi^{2}} \ln \left(\frac{m_{1}^{2}}{\mu^{2}}\right), \text { (B5) } \\
& \bar{B}\left(m_{1}^{2}, m_{2}^{2}, p^{2}\right)=\bar{J}\left(p^{2}\right)+\frac{\bar{A}\left(m_{1}^{2}\right)-\bar{A}\left(m_{2}^{2}\right)}{m_{1}^{2}-m_{2}^{2}}, \\
& \lambda_{0}=\frac{2}{\epsilon}+\ln (4 \pi)+1-\gamma .
\end{aligned}
$$


$\bar{J}\left(p^{2}\right)$ is defined in [6]. Three point functions $C_{1}, C_{2}, \ldots C_{222}$ can be written in rather simple form for $q^{2}=0$.

$$
\begin{aligned}
C_{1}= & \frac{Q^{2}+m_{1}^{2}-m_{1}^{2}}{2 q \cdot W} C_{0}+\frac{1}{2 q \cdot W}\left[B\left(m_{1}^{2}, m_{2}^{2}, W^{2}\right)-B\left(m_{1}^{2}, m_{1}^{2}, q^{2}\right)\right] \\
& -\frac{Q^{2}}{2(q \cdot W)^{2}}\left[B\left(m_{1}^{2}, m_{2}^{2}, W^{2}\right)-B\left(m_{1}^{2}, m_{2}^{2}, Q^{2}\right)\right] \\
C_{2}= & \frac{1}{2 q \cdot W}\left[B\left(m_{1}^{2}, m_{2}^{2}, W^{2}\right)-B\left(m_{1}^{2}, m_{2}^{2}, Q^{2}\right)\right] \\
C_{00}= & \frac{\lambda_{0}}{64 \pi^{2}}+\frac{1}{64 \pi^{2}}+\frac{1}{2} m_{1}^{2} C_{0}+\frac{Q^{2}}{4 q \cdot W}\left[\bar{B}_{1}\left(m_{1}^{2}, m_{2}^{2}, Q^{2}\right)-\bar{B}_{1}\left(m_{1}^{2}, m_{2}^{2}, W^{2}\right)\right] \\
C_{22}= & \frac{1}{2 q \cdot W}\left[B_{1}\left(m_{1}^{2}, m_{2}^{2}, W^{2}\right)-B_{1}\left(m_{1}^{2}, m_{2}^{2}, Q^{2}\right)\right] \\
C_{12}= & \frac{1}{2 q \cdot W}\left[B_{1}\left(m_{1}^{2}, m_{2}^{2}, W^{2}\right)+\left(Q^{2}+m_{1}^{2}-m_{2}^{2}\right) C_{2}-C_{00}-Q^{2} C_{22}\right] \\
C_{222}= & \frac{1}{2 q \cdot W}\left[B_{21}\left(m_{1}^{2}, m_{2}^{2}, W^{2}\right)-B_{21}\left(m_{1}^{2}, m_{2}^{2}, Q^{2}\right)\right] \\
C_{002}= & \frac{1}{2 q \cdot W}\left[B_{22}\left(m_{1}^{2}, m_{2}^{2}, W^{2}\right)-B_{22}\left(m_{1}^{2}, m_{2}^{2}, Q^{2}\right)\right] \\
C_{122}= & \frac{1}{2 q \cdot W}\left[B_{1}\left(m_{1}^{2}, m_{2}^{2}, W^{2}\right)-B_{21}\left(m_{1}^{2}, m_{2}^{2}, W^{2}\right)\right]-\frac{1}{q \cdot W} C_{002}, \\
C_{001}= & \frac{\lambda_{0}}{192 \pi^{2}}+\frac{1}{192 \pi^{2}}+\frac{1}{2} m_{1}^{2} C_{1}-\frac{1}{2} Q^{2} \bar{C}_{122}+\frac{1}{2} \bar{B}_{1}\left(m_{1}^{2}, m_{2}^{2}, W^{2}\right)-\frac{1}{2} \bar{B}_{21}\left(m_{1}^{2}, m_{2}^{2}, W^{2}\right), \\
C_{112}= & \frac{1}{2 q \cdot W}\left[B_{21}\left(m_{1}^{2}, m_{2}^{2}, W^{2}\right)-2 B_{1}\left(m_{1}^{2}, m_{2}^{2}, W^{2}\right)+B\left(m_{1}^{2}, m_{2}^{2}, W^{2}\right)\right]-\frac{2}{q \cdot W} C_{001}
\end{aligned}
$$

[1] A. Alavi-Harati, et al., KTeV Collaboration, Phys. Rev. D 64, 112004 (2001).

[2] Alexopoulos, et al., KTeV Collaboration, Phys. Rev. D 71, 012001 (2005).

[3] A. Lai, et al., NA48 Collaboration, Phys. Lett B 605, 247 (2005).

[4] E. Fishbach and J. Smith, Phys. Rev. 184, 1645 (1969);

H. W. Fearing, E. Fishbach and J. Smith, Phys. Rev. Lett. 24, 189 (1970);

H. W. Fearing, E. Fishbach and J. Smith, Phys. Rev. D 2, 542 (1970).

[5] B. R. Holstein, Phys. Rev. D 41, 2829 (1990).

[6] J. Bijnens, G. Ecker and J. Gasser, Nucl. Phys. B 396, 81 (1993).

[7] J. Gasser, B. Kubis, N. Paver, M. Verbeni, Eur. Phys. J. C 40, 205 (2005).

[8] B. Kubis, E. H. Muller, J. Gasser and M. Schmid, Eur. Phys. J. C 50, 557 (2007).

[9] M. Moulson, hep-ex/0611057.

[10] J. Bijnens, hep-ph/0707.0419. 
[11] J. Gasser, H. Leutwyler, Ann. Phys. 158, 142 (1984);

J. Gasser, H. Leutwyler, Nucl. Phys. B 250, 465 (1985).

[12] F. E. Low, Phys. Rev. 110, 974 (1958).

[13] S. L. Adler and Y. Dothan, Phys. Rev. 151, 1267 (1966).

[14] E. Abouzaid et al., Phys. Rev. Lett. 99, 081803 (2007).

[15] S. Scherer, Adv. Nucl. Phys. 27, 277 (2002).

[16] J. Wess and B. Zumino, Phys. Lett. B 37, 95 (1971).

[17] E. Witten, Nucl. Phys. B 233, 422 (1983).

[18] J. Bijnens and P. Talavera, Nucl. Phys. B669 (2003) 341.

[19] G. P. Lepage, Journal of Comp. Phys. 27, 192 (1978).

[20] T. Hahn and M. Perez-Victoria, Comput. Phys. Commun. 118, 153 (1999).

[21] G. J. Oldenborgh and J. A. M. Vermaseren, Z. Phys. C 46, 425 (1990).

[22] S. Eidelman et al., Phys. Lett. B 592, 1 (2004).

[23] J. Bijnens, G. Ecker, J. Gasser, hep-ph/9411232. 